\title{
ANALYTIC RESULTS FOR ROOTS OF TWO IRRATIONAL FUNCTIONS IN ELASTIC WAVE PROPAGATION
}

\author{
L. M. BROCK ${ }^{1}$
}

(Received 14 November 1996)

\begin{abstract}
The velocities of Rayleigh surface waves and, when they exist, Stoneley interface waves can be obtained as the roots of two irrational functions. Here previous results are extended by using standard operations related to the Wiener-Hopf technique to provide expressions in quadrature for these roots.
\end{abstract}

\section{Problem description}

The propagation of sound in elastic solids in the form of body waves whose speeds are defined in terms of material constants is well-established [9]. The transmission/reflection of such waves at material surfaces and interfaces has also been studied [3], and such studies have suggested the possibility of another type of wave - the Rayleigh wave - that propagates on a stress-free surface in such a manner that its effects are confined to the immediate vicinity of the surface [1]. As outlined in [1], it can be shown that the phase velocity $v$ of a Rayleigh wave along the planar surface of a homogeneous isotropic linearly-elastic solid must satisfy the equation

$$
\left(2 s^{2}-b^{2}\right)^{2}-4 s^{2} \sqrt{s^{2}-a^{2}} \sqrt{s^{2}-a^{2}}=0,
$$

where

$$
s=1 / v, \quad a=1 / v_{d}, \quad b=1 / v_{r}
$$

and $\left(v_{r}, v_{d}\right)$ are, respectively, the rotational and dilatational elastic body wave speeds given by

$$
v_{r}=\sqrt{\frac{\mu}{\rho}}, \quad v_{d}=\sqrt{2 \frac{1-v}{1-2 \nu}} v_{r} .
$$

${ }^{1}$ Department of Mechanical Engineering, University of Kentucky, Lexington, Kentucky 40506, USA

(C) Australian Mathematical Society, 1998, Serial-fee code 0334-2700/98 
The inverse velocity $s$, or slowness, is often the convenient quantity to work with. Here $(\mu, \rho, \nu)$ are, respectively, the shear modulus, mass density and Poisson's ratio for the elastic half-space. For an isotropic homogeneous solid, these constants are positive and real, with $0<v<0.5$ [12], so that we always have

$$
v_{d}>v_{r} \quad(b>a) .
$$

The fact that (1) exhibits the phase velocity and not also a wave-number indicates that Rayleigh waves are non-dispersive [10].

Stoneley [14] considered the analogous problem for a wave propagating along the interface of two welded (that is, continuity of both displacement and stress exists) dissimilar homogeneous isotropic linearly-elastic solids in such a manner that its effects are confined to the immediate vicinity of the interface. As described by Cagniard [6], it can be shown that the phase velocity $v$ of such a Stoneley wave for a planar interface must satisfy the equation

$$
\begin{aligned}
& s^{2}\left[\sqrt{s^{2}-a_{1}^{2}} \sqrt{s^{2}-b_{1}^{2}} \sqrt{s^{2}-a_{2}^{2}} \sqrt{s^{2}-b_{2}^{2}}+\left(\Omega_{1}+\Omega_{2}-s^{2}\right)^{2}\right] \\
& -\left(\Omega_{2}-s^{2}\right)^{2} \sqrt{s^{2}-a_{1}^{2}} \sqrt{s^{2}-b_{1}^{2}}-\left(\Omega_{1}-s^{2}\right)^{2} \sqrt{s^{2}-a_{2}^{2}} \sqrt{s^{2}-b_{2}^{2}} \\
& \quad+\Omega_{1} \Omega_{2}\left(\sqrt{s^{2}-a_{1}^{2}} \sqrt{s^{2}-b_{2}^{2}}+\sqrt{s^{2}-a_{2}^{2}} \sqrt{s^{2}-b_{1}^{2}}\right)=0,
\end{aligned}
$$

where the subscripts $(1,2)$ refer to the two welded solids and

$$
\Omega_{1}=c_{1} b_{1}^{2}, \quad \Omega_{2}=c_{2} b_{2}^{2}, \quad c_{1}=\frac{1}{2}-c_{2}=\frac{\mu_{1}}{2\left(\mu_{2}-\mu_{1}\right)} .
$$

Equation (2a) holds again, therefore as do, with appropriate subscripts attached, (2b,c) and (3). The absence of a wave-number in (5) indicates that Stoneley waves are also non-dispersive.

\section{Previous results}

To obtain information about possible (real) solutions of (1), one can study [1] the roots of the well-known Rayleigh function

$$
R(z)=4 z^{2} \alpha \beta+T^{2}
$$

in the complex $z$-plane. Here

$$
T=b^{2}-2 z^{2}, \quad \alpha=\sqrt{a^{2}-z^{2}}, \quad \beta=\sqrt{b^{2}-z^{2}}
$$


and $R(z)$ is rendered single-valued by introducing branch cuts for $(\alpha, \beta)$ along $\operatorname{Im}(z)=$ $0,|\operatorname{Re}(z)|>a$ and $\operatorname{Im}(z)=0,|\operatorname{Re}(z)|>b$, respectively, such that $\operatorname{Re}(\alpha, \beta) \geq 0$ everywhere in the cut $z$-plane. Application of the standard argument principle [7] then shows that $R(z)$ always exhibits the isolated real roots $z= \pm s_{0}$ of order 1 , where $s_{0}$ lies in the range

$$
s_{0}>b \text {. }
$$

Analogously [6], for information about (real) solutions to (5) the Stoneley function

$$
S(z)=z^{2}\left(\alpha_{1} \beta_{1} \alpha_{2} \beta_{2}+P_{12}^{2}\right)+\Omega_{1} \Omega_{2}\left(\alpha_{1} \beta_{2}+\alpha_{2} \beta_{1}\right)-P_{2}^{2} \alpha_{1} \beta_{1}-P_{1}^{2} \alpha_{2} \beta_{2}
$$

can be introduced, where $(8 \mathrm{~b}, \mathrm{c})$ hold again with appropriate subscripts, and

$$
P_{1}=\Omega_{1}-z^{2}, \quad P_{2}=\Omega_{2}-z^{2}, \quad P_{12}=\Omega_{1}+\Omega_{2}-z^{2} .
$$

In this case, the physical restrictions (4) on body wave speeds allow for a given pair of welded materials the six possible combinations

$$
\begin{aligned}
& b_{2}>a_{2}>b_{1}>a_{1}, \\
& b_{2}>b_{1}>a_{2}>a_{1}, \\
& b_{2}>b_{1}>a_{1}>a_{2}, \\
& b_{1}>b_{2}>a_{1}>a_{2}, \\
& b_{1}>b_{2}>a_{2}>a_{1}, \\
& b_{1}>a_{1}>b_{2}>a_{2},
\end{aligned}
$$

so that the function $S(z)$ can be made analytic in the $z$-plane cut along $\operatorname{Im}(z)=0$, $\min \left(a_{1}, a_{2}\right)<|\operatorname{Re}(z)|<\max \left(b_{1}, b_{2}\right)$ by requiring that $\operatorname{Re}\left(\alpha_{1}, \beta_{1}, \alpha_{2}, \beta_{2}\right) \geq 0$ in that cut plane. While (4) precludes the limit cases $\left(b_{1}=a_{1}, b_{2}=a_{2}\right)$, it is conceivable that the two dissimilar welded solids would be matched in other particulars. In this brief demonstration, however, we will treat the general situation, that is, no partial material matching occurs, so that the strict inequalities in (12) are maintained, and $\Omega_{1} \neq \Omega_{2}$.

Then, application of the argument principle [7] shows that $S(z)$ has the isolated real roots $z= \pm s_{0}$ of order 1 in the range

$$
s_{0}>\max \left(b_{1}, b_{2}\right)
$$

only if the welded material properties are such that

$$
\begin{gathered}
\sqrt{b_{1}^{2}-b_{2}^{2}}\left[\Omega_{1} \Omega_{2} \sqrt{b_{1}^{2}-a_{2}^{2}}-\left(\Omega_{1}-b_{1}^{2}\right)^{2} \sqrt{b_{1}^{2}-a_{2}^{2}}\right] \\
+b_{1}^{2}\left(\Omega_{1}+\Omega_{2}-b_{1}^{2}\right)^{2}>0 \quad\left(b_{1}>b_{2}\right), \\
\sqrt{b_{2}^{2}-b_{1}^{2}}\left[\Omega_{2} \Omega_{1} \sqrt{b_{2}^{2}-a_{1}^{2}}-\left(\Omega_{2}-b_{2}^{2}\right)^{2} \sqrt{b_{2}^{2}-a_{1}^{2}}\right] \\
+b_{2}^{2}\left(\Omega_{2}+\Omega_{1}-b_{2}^{2}\right)^{2}>0 \quad\left(b_{2}>b_{1}\right) .
\end{gathered}
$$


If (14) is not satisfied, then $S(z)$ has no roots in the cut $z$-plane.

Clearly, the values of $s_{0}$ for $(R, S)$ can be found for any given set(s) of values $(\mu, \rho, v)$, see, for example, $[1,6]$. Indeed, in the case of $R,(7)$ can be rationalized to a cubic equation in $z^{2}$, although two of the roots of that equation are extraneous. In what follows, expressions in quadrature for $s_{0}$ are obtained analytically for both $(R, S)$ by the application of standard operations.

\section{Results for $R$}

Study of (7) shows that $R$ is holomorphic in the $z$-plane cut along $\operatorname{Im}(z)=0$, $a<|\operatorname{Re}(z)|<b$, and behaves as

$$
R \approx 2\left(a^{2}-b^{2}\right) z^{2}, \quad|z| \rightarrow \infty .
$$

Therefore, one can introduce the function

$$
G(z)=\frac{R(z)}{2\left(a^{2}-b^{2}\right)\left(z^{2}-s_{0}^{2}\right)}
$$

which is also holomorphic in this cut plane, but exhibits no zeros there and approaches unity at infinity. It is therefore possible $[1,10,13]$ to write $G$ as the product of functions $G_{ \pm}$which are holomorphic in, respectively, the overlapping halves $\operatorname{Re}(z)>-a$ and $\operatorname{Re}(z)<a$ of the complex $z$-plane. Specifically, performing factorization operations formulated by Noble [10] for the well-known Wiener-Hopf technique gives

$$
\ln G_{ \pm}(z)=-\frac{1}{\pi} \int_{a}^{b} \tan ^{-1} \frac{4 u^{2} A \beta}{T^{2}} \frac{d u}{u \pm z}, A=\sqrt{u^{2}-a^{2}}, B=\sqrt{u^{2}-b^{2}}
$$

where integration is along the positive $\operatorname{Re}(u)$-axis. At $z=0$ the functions $R$ and $G_{ \pm}$ are holomorphic, and $G_{-}=G_{+}$. Therefore, replacing the left-hand side of (16) with $G_{+} G_{-}$and evaluating the result at $z=0$ gives, in view of (7), the explicit formula

$$
s_{0}=\frac{b^{2}}{\sqrt{2\left(b^{2}-a^{2}\right)}} \frac{1}{G_{+}(0)}
$$

for the root of $R$.

\section{Results for $S$}

The procedure for $S(z)$ when (14) holds follows by analogy. That is, a function

$$
G(z)=\frac{S(z)}{4\left(4 \Omega_{1}-a_{1}^{2}-b_{1}^{2}\right)\left(4 \Omega_{2}-a_{2}^{2}-b_{2}^{2}\right)\left(z^{2}-s_{0}^{2}\right)}
$$


is introduced that is holomorphic and exhibits no zeros in the $z$-plane cut along $\operatorname{Im}(z)=$ $0, \min \left(a_{1}, a_{2}\right)<|\operatorname{Re}(z)|<\max \left(b_{1}, b_{2}\right)$, and approaches unity at infinity. The same factorization procedure [11] used for (16) then yields $G$ as the product of functions $G_{ \pm}$that are analytic in the overlapping halves, respectively, $\operatorname{Re}(z)>-\min \left(a_{1}, a_{2}\right)$ and $\operatorname{Re}(z)<\min \left(a_{1}, a_{2}\right)$ of the complex $z$-plane. Equations (12) and (14) lead to, however, six different forms for $G_{ \pm}$. It can be shown that when (14a) governs, then

$$
\begin{aligned}
& \ln G_{ \pm}(z)=-\frac{1}{\pi}\left(\int_{a_{1}}^{b_{1}} C_{1}+\int_{b_{1}}^{a_{2}} D_{1}+\int_{a_{2}}^{b_{2}} E_{1}\right) \frac{d u}{u \pm z}, \\
& \ln G_{ \pm}(z)=-\frac{1}{\pi}\left(\int_{a_{1}}^{a_{2}} C_{1}+\int_{a_{2}}^{b_{1}} D_{2}+\int_{b_{1}}^{b_{2}} E_{1}\right) \frac{d u}{u \pm z} \\
& \ln G_{ \pm}(z)=-\frac{1}{\pi}\left(\int_{a_{2}}^{a_{1}} C_{3}+\int_{a_{1}}^{b_{1}} D_{2}+\int_{b_{1}}^{b_{2}} E_{1}\right) \frac{d u}{u \pm z}
\end{aligned}
$$

for the cases (12a-c), respectively, while when (14b) governs, the formulas

$$
\begin{aligned}
& \ln G_{ \pm}(z)=-\frac{1}{\pi}\left(\int_{a_{2}}^{a_{1}} C_{3}+\int_{a_{1}}^{b_{2}} D_{2}+\int_{b_{2}}^{b_{1}} E_{4}\right) \frac{d u}{u \pm z}, \\
& \ln G_{ \pm}(z)=-\frac{1}{\pi}\left(\int_{a_{1}}^{a_{2}} C_{1}+\int_{a_{2}}^{b_{2}} D_{2}+\int_{b_{2}}^{b_{1}} E_{4}\right) \frac{d u}{u \pm z}, \\
& \ln G_{ \pm}(z)=-\frac{1}{\pi}\left(\int_{a_{2}}^{b_{2}} C_{3}+\int_{b_{2}}^{a_{1}} D_{6}+\int_{a_{1}}^{b_{1}} E_{4}\right) \frac{d u}{u \pm z}
\end{aligned}
$$

hold for the cases (12d-f), respectively. In (20) the relations

$$
\begin{aligned}
& C_{1}=\tan ^{-1} A_{1} \frac{u^{2} \beta_{1} \alpha_{2} \beta_{2}-\Omega_{1} \Omega_{2} \beta_{2}+\beta_{1} P_{2}^{2}}{\Omega_{1} \Omega_{1} \alpha_{2} \beta_{1}-\alpha_{2} \beta_{2} P_{1}^{2}-u^{2} P_{12}^{2}}, \\
& C_{3}=\tan ^{-1} A_{2} \frac{u^{2} \beta_{2} \alpha_{1} \beta_{1}-\Omega_{1} \Omega_{2} \beta_{1}+\beta_{2} P_{1}^{2}}{\Omega_{1} \Omega_{1} \alpha_{1} \beta_{2}-\alpha_{1} \beta_{1} P_{2}^{2}-u^{2} P_{12}^{2}}, \\
& D_{1}=\tan ^{-1} \Omega_{1} \Omega_{2} \frac{\alpha_{2} B_{1}+A_{1} \beta_{2}}{\alpha_{2} \beta_{2} P_{1}^{2}+u^{2}\left(P_{12}^{2}-A_{1} B_{1} \alpha_{2} \beta_{2}\right)}, \\
& D_{2}=\tan ^{-1} \frac{1}{u^{2}} \frac{A_{1}\left(\Omega_{1} \Omega_{2} \beta_{2}-\beta_{1} P_{2}^{2}\right)+A_{2}\left(\Omega_{1} \Omega_{2} \beta_{1}-\beta_{2} P_{1}^{2}\right)}{P_{12}^{2}-A_{1} \beta_{1} A_{2} \beta_{2}}, \\
& D_{6}=\tan ^{-1} \Omega_{1} \Omega_{2} \frac{A_{2} \beta_{1}+\alpha_{1} B_{2}}{\alpha_{1} \beta_{1} P_{2}^{2}+u^{2}\left(P_{12}^{2}-\alpha_{1} \beta_{1} A_{2} B_{2}\right)}, \\
& E_{1}=\tan ^{-1} \beta_{2} \frac{u^{2} A_{1} B_{1} A_{2}+\Omega_{1} \Omega_{2} A_{1}-A_{2} P_{1}^{2}}{\Omega_{1} \Omega_{2} A_{2} B_{1}-A_{1} B_{1} P_{2}^{2}+u^{2} P_{12}^{2}}, \\
& E_{4}=\tan ^{-1} \beta_{1} \frac{u^{2} A_{1} A_{2} B_{2}+\Omega_{1} \Omega_{2} A_{2}-A_{1} P_{2}^{2}}{\Omega_{1} \Omega_{2} A_{1} B_{2}-A_{2} B_{2} P_{1}^{2}+u^{2} P_{12}^{2}}
\end{aligned}
$$


TABLE 1.

$\begin{array}{lcccc} & \mu(\mathrm{GPa}) & \rho\left(\mathrm{kg} / \mathrm{m}^{3}\right) & \nu & v(\mathrm{~m} / \mathrm{s}, \text { Rayleigh }) \\ \text { aluminium } & 25.9 & 2768 & 0.33 & 2856 \\ \text { brass } & 39.3 & 8145 & 0.35 & 2019 \\ \text { copper } & 42.7 & 8941 & 0.35 & 2043 \\ \text { steel } & 73.1 & 7778 & 0.3 & 2841 \\ \text { titanium } & 41.4 & 4512 & 0.34 & 2825\end{array}$

and $(17 \mathrm{~b}, \mathrm{c})$ apply, with appropriate subscripts attached. In (20) integration is over the positive $\operatorname{Re}(u)$-axis. By evaluating (19) at $z=0$, where $G_{ \pm}$and $S$ are all holomorphic and $G_{-}=G_{+}$, the explicit result

$$
s_{0}=\sqrt{\frac{2\left(\Omega_{2} b_{1}-\Omega_{1} b_{2}\right)\left(\Omega_{2} a_{1}-\Omega_{1} a_{2}\right)}{\left(4 \Omega_{1}-a_{1}^{2}-b_{1}^{2}\right)\left(4 \Omega_{2}-a_{2}^{2}-b_{2}^{2}\right)}} \frac{1}{G_{+}(0)}
$$

can be obtained when, of course, (14) holds. In view of (3), (4), (6) and (12), the argument of the radical in (22) is always positive.

\section{Numerical values}

The expression (18) was checked against a table of values [1] for the Rayleigh wave phase velocity and found to agree to the three significant figures given. Then (18) and (22) were used to calculate Rayleigh and Stoneley phase velocity values for various standard [2] engineering materials, and those values were compared with the roots of, respectively, (1) and (5). The integrations arising in (18) and (22) were performed by standard Gaussian quadrature [8], while the roots of (1) and (5) were obtained by a standard [8] false position iterative scheme. The values obtained agreed to four significant figures. A list of Rayleigh values is given in Table 1 for five materials, along with the corresponding (typical) values $(\mu, \rho, v)$. Table 2 then lists the corresponding Stoneley values for those combinations of welded materials in Table 1 for which Stoneley waves exist, that is (14) holds, along with the corresponding Rayleigh values. It is noted that the Stoneley value is in each case larger than the Rayleigh values for either of the welded materials.

\section{Comments}

It should be noted that the empirical formulas for the Rayleigh/Stoneley wave phase velocity can readily be derived from plots of numerically-determined values, 
TABLE 2.

$\begin{array}{lccc} & v(\mathrm{~m} / \mathrm{s}, \text { Rayleigh-1) } & v(\mathrm{~m} / \mathrm{s}, \text { Rayleigh-2) } & v(\mathrm{~m} / \mathrm{s}, \text { Stoneley }) \\ \begin{array}{l}\text { aluminium-1 } \\ \text { steel-2 }\end{array} & 2856 & 2841 & 3025 \\ \begin{array}{l}\text { aluminium-1 } \\ \text { titanium-2 }\end{array} & 2856 & 2825 & 3027\end{array}$

for example [1]. Indeed, such formulas are simpler than (18) or (22). Moreover, the numerical integrations in (18) and (22) required more computational time than the numerical root-finding schemes used for (1) and (5).

However, (18) and (22) are exact, and allow a more analytic study of the dependence of Rayleigh/Stoneley velocities upon material properties to be made, especially in the Stoneley case when the partial material matching neglected here arises. Moreover, the procedure used to develop these formulas demonstrates that certain roots of two physically-relevant irrational functions can be obtained analytically. This procedure has been extended $[4,5]$ to cases of homogeneous isotropic linear coupled thermoelastic materials in which, due to the coupling between the mechanical and temperature fields, the Rayleigh/Stoneley signals are dispersive, that is, the phase velocity depends upon wave number.

\section{Acknowledgment}

This work was supported in part by NSF Grant DMS 9121700.

\section{References}

[1] J. D. Achenbach, Wave propagation in elastic solids (North-Holland/American Elsevier, New-York, 1973).

[2] H. E. Boyer and T. L. Gall, Metals Handbook (American Society for Metals, 1985).

[3] L. M. Brekhovskikh, Waves in layered media (D. Liberman, translator) (Academic Press, New York, 1960).

[4] L. M. Brock, 'Transient thermal response near dynamically-loaded cracks during dislocation generation', Acta Mechanica 110 (1995) 199-216.

[5] L. M. Brock, 'Transient three-dimentional Rayleigh and Stoneley signal effects in thermoelastic solids', Intemational J. of Solids and Structures, 34 (1997) 1463-1478.

[6] L. Cagniard, Reflection and refraction of progressive seismic waves (E. A. Flinn and C. H. Dix, translators) (McGraw-Hill, New York, 1962).

[7] E. Hille, Analytic function theory, Volume I (Ginn/Blaisdell, Waltham (MA), 1959).

[8] K. S. Kunz, Numerical analysis (McGraw-Hill, New York, 1957). 
[9] A. E. H. Love, A treatise on the mathematical theory of elasticity (Dover, New York, 1944).

[10] P. M. Morse and H. Feshbach, Methods of theoretical physics, Part I (McGraw-Hill, New York, 1953).

[11] R. Noble, Methods based on the Wiener-Hopf technique (Pergamon, New York, 1958).

[12] I. S. Sokolnikoff, Mathematical theory of elasticity (McGraw-Hill, New York, 1956).

[13] I. Stakgold, Boundary value problems of mathematical physics, Volume II (MacMillan, New York, 1967).

[14] R. Stoneley, 'Elastic waves at the surface of separation of two solids', Proceedings of the Royal Society (London) A106 (1924) 416-418. 